
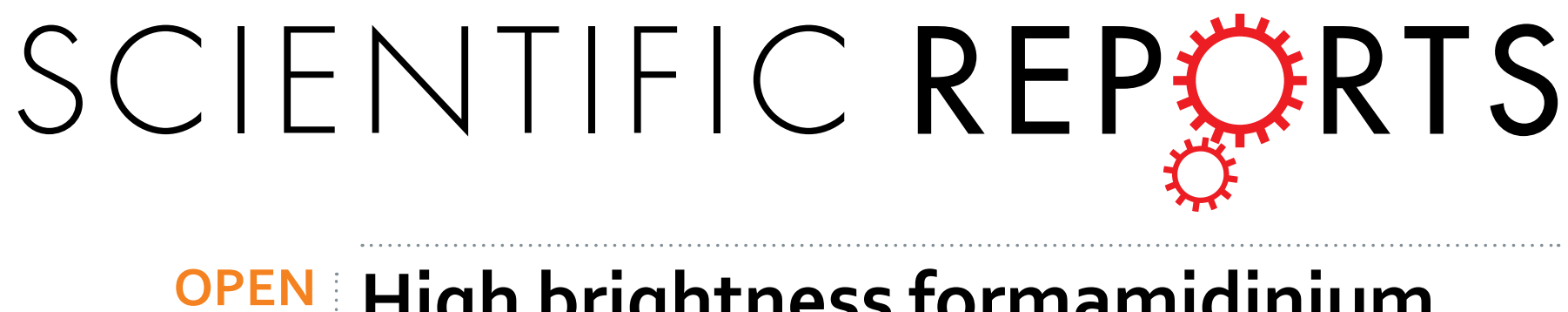

\title{
High brightness formamidinium lead bromide perovskite nanocrystal light emitting devices
}

Received: 21 June 2016

Accepted: 10 October 2016

Published: 09 November 2016
Ajay Perumal ${ }^{1}$, Sushant Shendre ${ }^{1}$, Mingjie $\mathrm{Li}^{2}$, Yong Kang Eugene Tay ${ }^{2}$, Vijay Kumar Sharma ${ }^{1,2,3}$, Shi Chen ${ }^{2}$, Zhanhua Wei ${ }^{2}$, Oing Liu ${ }^{4}$, Yuan Gao ${ }^{1}$, Pio John S. Buenconsejo ${ }^{4}$, Swee Tiam Tan ${ }^{1}$, Chee Lip Gan ${ }^{4}$, Qihua Xiong ${ }^{2}$, Tze Chien Sum ${ }^{2}$ \& Hilmi Volkan Demir ${ }^{1,2,3}$

Formamidinium lead halide $\left(\mathrm{FAPbX}_{3}\right)$ has attracted greater attention and is more prominent recently in photovoltaic devices due to its broad absorption and higher thermal stability in comparison to more popular methylammonium lead halide $\mathrm{MAPbX}_{3}$. Herein, a simple and highly reproducible room temperature synthesis of device grade high quality formamidinium lead bromide $\mathrm{CH}\left(\mathrm{NH}_{2}\right)_{2} \mathrm{PbBr}_{3}$ ( $\mathrm{FAPbBr}_{3}$ ) colloidal nanocrystals (NC) having high photoluminescence quantum efficiency (PLOE) of $55-65 \%$ is reported. In addition, we demonstrate high brightness perovskite light emitting device (Pe-LED) with these $\mathrm{FAPbBr}_{3}$ perovskite NC thin film using 2, $2^{\prime}, 2^{\prime \prime}$-(1,3,5-Benzinetriyl)-tris(1-phenyl-1$\mathrm{H}$-benzimidazole) commonly known as TPBi and 4,6-Bis(3,5-di(pyridin-3-yl)phenyl)-2-methylpyrimidine (B3PYMPM) as electron transport layers (ETL). The Pe-LED device with B3PYMPM as ETL has bright electroluminescence of up to $2714 \mathrm{~cd} / \mathrm{m}^{2}$, while the Pe-LED device with TPBi as ETL has higher peak luminous efficiency of $6.4 \mathrm{~cd} / \mathrm{A}$ and peak luminous power efficiency of $5.7 \mathrm{Im} / \mathrm{W}$. To our knowledge this is the first report on high brightness light emitting device based on $\mathrm{CH}\left(\mathrm{NH}_{2}\right)_{2} \mathrm{PbBr}_{3}$ widely known as $\mathrm{FAPbBr}_{3}$ nanocrystals in literature.

Lead halide based perovskite materials have remarkable optoelectronic properties like high absorption coefficient, large mobility and easier bandgap tunability ${ }^{1-8}$. There is an exponential surge in interest, in these class of materials especially in photovoltaic devices ${ }^{6,9-13}$. The semiconducting lead halide based perovskite system in particular the organic-inorganic hybrid system, namely methyl ammonium lead halide $\mathrm{CH}_{3} \mathrm{NH}_{3} \mathrm{PbBr}_{3}\left(\mathrm{MAPbX}_{3}\right)^{11-13}$ has shown great promise and photovoltaic devices with power conversion efficiency in excess of $20 \%$ have been reported ${ }^{14,15}$. Recently, formamidinium lead halide $\mathrm{CH}\left(\mathrm{NH}_{2}\right)_{2} \mathrm{PbX}_{3}\left(\mathrm{FAPbX}_{3}\right)$ perovskite ${ }^{16,17}$ has gained prominence with higher thermal stability in comparison to more popular methylammonium lead halide perovskite and photovoltaic devices with power conversion efficiencies in excess of $20 \%$ have been reported with $\mathrm{FAPbI}_{3}{ }^{15}$.

Besides their application in photovoltaic devices the organic-inorganic lead halide based hybrid perovskites have shown excellent optoelectronic properties for their application in passive photo luminescent or active electroluminescent optoelectronic devices due to their large absorption coefficients, almost no defects, and reduced Auger recombination ${ }^{6,18-31}$. High quality polycrystalline films can be easily processed at room temperature in ambient via simple solution based techniques and does not require any high temperature processing steps ${ }^{6-8,32}$. The emission wavelength of the hybrid perovskite material can be tuned over the entire visible to infrared region of the electromagnetic spectrum by simple halide cation exchange or by inter mixing of halide cations, making them an excellent class of materials especially for their use in low-cost and large area optoelectronic device related applications $\mathrm{s}^{3,21,33}$. As early as 1990s, the long-chain layered 2 dimensional alkyl organic/inorganic lead halide perovskite $\left(\mathrm{C}_{6} \mathrm{H}_{5} \mathrm{C}_{2} \mathrm{H}_{4} \mathrm{NH}_{3}\right)_{2} \mathrm{PbI}_{4}{ }^{34,35}$ was used in LED devices operating at liquid-nitrogen temperatures. Later, perovskite LED device was also demonstrated with $\left(\mathrm{H}_{3} \mathrm{NC}_{2} \mathrm{H}_{4} \mathrm{C}_{16} \mathrm{H}_{8} \mathrm{~S}_{4} \mathrm{C}_{2} \mathrm{H}_{4} \mathrm{NH}_{3}\right) \mathrm{PbX}_{4}(\mathrm{X}=\mathrm{Cl}, \mathrm{Br}$, I $)$ which operates

${ }^{1}$ LUMINOUS! Center of Excellence for Semiconductor Lighting and Displays, School of Electrical and Electronic Engineering, Nanyang Technological University, 639798, Singapore. ${ }^{2}$ Division of Physics and Applied Physics, School of Physical and Mathematical Sciences, Nanyang Technological University, 637371, Singapore. ${ }^{3}$ UNAM-Institute of Materials Science and Nanotechnology, Department of Electrical and Electronics Engineering, Department of Physics, Bilkent University, Ankara, 06800, Turkey. ${ }^{4}$ School of Materials Science and Engineering, Nanyang Technological University, 639798, Singapore. Correspondence and requests for materials should be addressed to A.P. (email: ajay@ntu.edu.sg) or H.V.D. (email: volkan@ntu.edu.sg) 


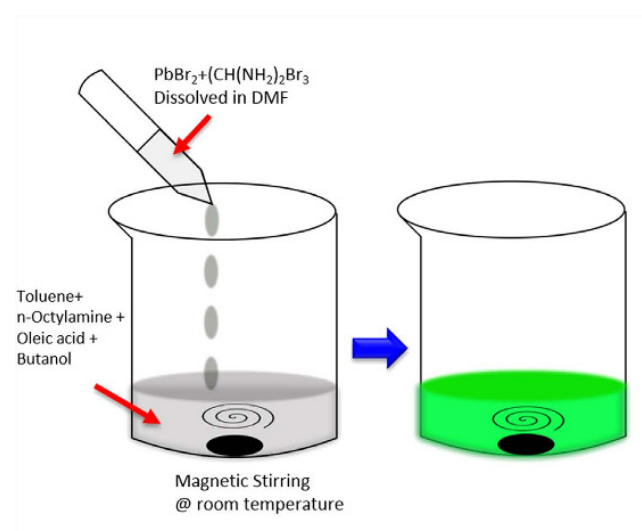

(a)

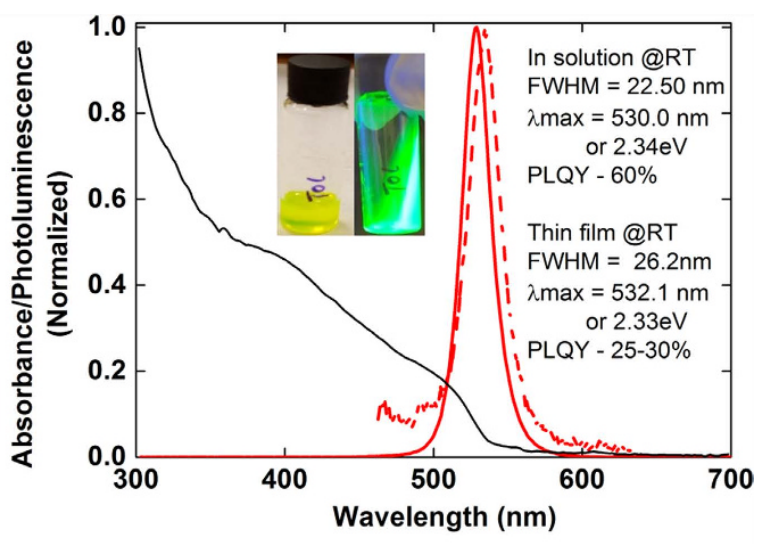

(b)

Figure 1. (a) Brief schematic illustration of the synthesis procedure where the precursor consisting of $\mathrm{PbBr}_{2}$ and $\mathrm{CH}\left(\mathrm{NH}_{2}\right)_{2} \mathrm{Br}$ dissolved in DMF solvent is added dropwise to toluene consisting of ligands and stabilizer under vigorous stirring at room temperature. (b) The absorption and PL spectra of $\mathrm{FAPbBr}_{3}$ nanocrystals in toluene solution, for reference the thin film PL is also overlaid on the solution PL. The inset shows $\mathrm{FAPbBr}_{3} \mathrm{NC}$ in toluene under ambient light and under UV exposure.

at room temperature with power efficiency of $0.1 \mathrm{~lm} / \mathrm{W}^{36}$. Friend's group reported infrared emitting LED devices with low turn on voltage $(1.5 \mathrm{~V})$ and high external quantum efficiency (EQE) of $0.76 \%$ by using short chain perovskite material $\mathrm{MAPbX}_{3}$ as an active layer ${ }^{25}$. More recently, Tae Woo Lee and co-workers have demonstrated thin film perovskite green emitting LED device with EQE of $8.5 \%$ and power efficiency of $42.9 \mathrm{~cd} / \mathrm{A}$ with $\mathrm{MAPbBr}_{3}$ thin films, demonstrating the potential of perovskites in high efficiency LED devices ${ }^{37}$. Their potential application in lasing ${ }^{38-40}$ and light emitting transistor (LET) ${ }^{41}$ have also been reported.

Recently, a lot of progress has been made in the synthesis of perovskite nanocrystals (NCs) adapting similar synthesis procedures to that of inorganic semiconductor nanocrystals. Perovskite NCs exhibiting high photoluminescence quantum efficiency (PLQE) and high color purity have been reported ${ }^{22,42-44}$. The quantum confinement effects, in perovskite nanostructures are not as prominent as in inorganic semiconductor nanocrystals ${ }^{43,45,46}$. The NC size variation does not result in tuning of emission wavelength over entire visible range of the electromagnetic spectrum. The color tuning is much more pronounced and controlled in perovskite NCs via cation replacement or intermixing of the cation with different halides ${ }^{3,33,43,44}$. Previously, methyl ammonium lead halide and cesium lead halide based nanocrystals have been employed for fabricating light-emitting devices ${ }^{19,20,24,47}$.

Herein, we report a simple, easy to perform and highly reproducible room temperature synthesis of device grade, polycrystalline and highly luminescent $\mathrm{FAPbBr}_{3}$ colloidal NC having a high PLQE of 55-65\%. These perovskite NC's exhibit bright photoluminescence (PL) with an emission peak centred at $531.3 \mathrm{~nm}$ having a very narrow full width at half maximum (FWHM) of $21.6 \mathrm{~nm}$. The PLQE value reported is 10 fold improvement in comparison to prior published literature for $\mathrm{FAPbBr}_{3} \mathrm{NP}$ synthesis (PLQE of 5\%), using them light emitting electrochemical cells were demonstrated with maximum light output of $1-2 \mathrm{~cd} / \mathrm{m}^{248}$. We demonstrate high brightness LEDs based on solution cast $\mathrm{FAPbBr}_{3}$ nanocrystals, and the Pe-LED devices have bright electroluminescence of up to $2714 \mathrm{~cd} / \mathrm{m}^{2}$, luminous efficiency of $6.4 \mathrm{~cd} / \mathrm{A}$ and luminous power efficiency of $5.7 \mathrm{~lm} / \mathrm{W}$. This is the first report on high brightness light emitting diode device based on $\mathrm{FAPbBr}_{3}$ nanocrystals and to our knowledge, till date there is no report on efficient Pe-LEDs using $\mathrm{FAPbBr}_{3} \mathrm{NCs}$ in literature. Also with temperature dependent exciton dynamics, we propose exciton quenching in perovskite nanocrystals especially the roll off in luminance and efficiency at higher voltages which limits the performance of perovskite LED devices.

\section{Results and Discussion}

The synthesis procedure for $\mathrm{FAPbBr}_{3} \mathrm{NCs}$ is similar to that of $\mathrm{MAPbBr}_{3}$ nanocrystals reported earlier by Feng Zhang and co-workers ${ }^{22}$. Briefly, a precursor solution consisting of $\mathrm{CH}\left(\mathrm{NH}_{2}\right)_{2} \mathrm{Br}$ and $\mathrm{PbBr}_{2}$ dissolved in $\mathrm{N}$-dimethylformamide (DMF) is added dropwise into a solution consisting of toluene and organic long chain ligands (n-Octylamine) and a stabilizer (oleic acid) under constant stirring. The transparent toluene solution with ligands and stabilizer turns to greenish yellow in color after the precursor addition, confirming the formation of colloidal perovskite nanocrystals as shown in Fig. 1(a).

The greenish yellow colloidal perovskite nanocrystal solution is centrifuged at $7840 \mathrm{rpm}$ for 5 minutes and the supernatant is discarded. The bottom precipitate is again re-dissolved in fresh anhydrous toluene and the solution is centrifuged again for 5 minutes at $7840 \mathrm{rpm}$, this time the supernatant is collected for device fabrication and the bottom precipitate is discarded. Figure 1(b) shows the absorption (black) and emission (red) spectra of $\mathrm{FAPbBr}_{3}$ NCs dispersed in toluene. The PL emission in toluene is roughly centred on the onset of absorption peak with PL peaked at $530.0 \mathrm{~nm}(2.34 \mathrm{eV})$ having a narrow full width half maximum (FWHM) of $22.5 \mathrm{~nm}$. The thin film PL emission at room temperature has PL emission peak at $532 \mathrm{~nm}$ which is slightly red shifted in comparison to solution PL emission peak and has FWHM of $26 \mathrm{~nm}$ slightly broader compared to solution FWHM. The PL emission is mainly due to exciton recombination in these materials as there is very little Stokes shift between absorption peak (first exciton peak) and the PL emission peak in perovskite NCs. The bulk $\mathrm{FAPbBr}_{3}$ films have been 

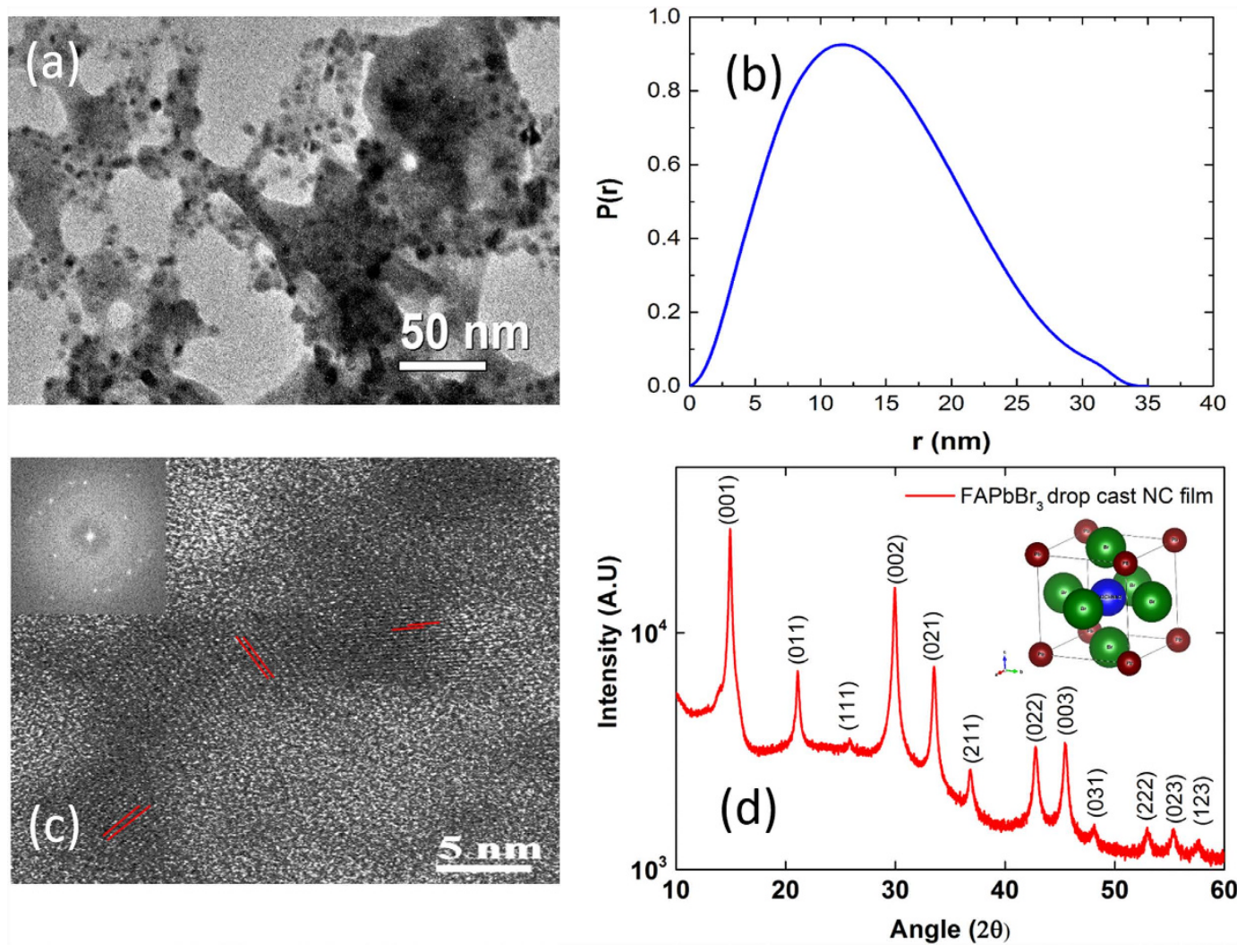

Figure 2. (a) Transmission electron microscopy (TEM) image of $\mathrm{FAPbBr}_{3} \mathrm{NCs}$. The black dots correspond to $\mathrm{FAPbBr}_{3}$ NCs having an average size of $10-15 \mathrm{~nm}$. (b) Particle size distributions measured via small angle $\mathrm{X}$-ray scattering technique (SAXS) the as synthesized $\mathrm{FAPbBr}_{3} \mathrm{NCs}$ with an average size of $12-14 \mathrm{~nm}$.

(c) The high-resolution transmission electron microscopy (HR-TEM) of the FAPbBr 3 NCs with its fast Fourier transformation (FFT) image shown in the inset. (d) X-ray diffraction (XRD) patterns for drop cast $\mathrm{FAPbBr}_{3}$ NCs.

reported to have PL emission peak $>550 \mathrm{~nm}^{16,17,49,50}$, hence the emission peak for $\mathrm{FAPbBr}_{3} \mathrm{NCs}$ is blue-shifted by $10-20 \mathrm{~nm}$ compared to that of $\mathrm{FAPbBr}_{3}$ bulk materials. The $\mathrm{FAPbBr}_{3} \mathrm{NC}$ solution in ambient light and under UV-excitation is shown in the inset of Fig. 1(b). Strong PL is observed from $\mathrm{FAPbBr}_{3} \mathrm{NCs}$ under UV light for both NCs in solution and in thin film form. The synthesis procedure is highly reproducible with PLQE in solution between 55-65\% for different batch synthesis of perovskites as shown in Supplementary info Figure S6.

The as-prepared perovskite NCs are primarily polycrystalline having broad size distribution. Figure 2(a)

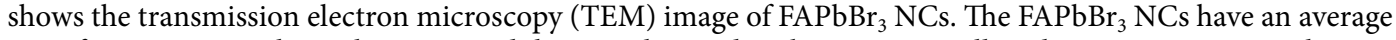
size of $10-15 \mathrm{~nm}$. We have also measured the particle size distribution via small angle $\mathrm{x}$-ray scattering technique and the as synthesized $\mathrm{FAPbBr}_{3} \mathrm{NCs}$ have particle distribution from 1-30 nm with an average size of $12-14 \mathrm{~nm}$ shown in Fig. 2(b). The average size obtained from SAXS are consistent with the TEM measurements. Further information as to how to formulate the particle size from SAXS is described in Supplementary Information. We can also resolve the crystalline lattice planes of these $\mathrm{FAPbBr}_{3} \mathrm{NCs}$ in the high resolution transmission electron microscopy (HR-TEM) image shown in (Fig. 2(c)) with its fast Fourier transformation (FFT) image shown in the inset of Fig. 2(c). The TEM measurements confirm high degree of crystallinity in these $\mathrm{FAPbBr}_{3} \mathrm{NCs}$. X-ray diffraction (XRD) pattern for drop cast $\mathrm{FAPbBr}_{3} \mathrm{NCs}$ is recorded at room temperature and the XRD spectra is shown in Fig. 2(d).

The XRD data clearly shows that the NCs are highly polycrystalline. The XRD patterns are indexed to the cubic phase of $\mathrm{FAPbBr}_{3}$ with a unit cell of $6.006 \AA$ with $\mathrm{Pm}-3 \mathrm{~m}$ space group, similar to that of bulk $\mathrm{FAPbBr}_{3}$ perovskite $^{17,51}$. The detailed indexing is shown in Table ST1 of supplementary info. The inter-planar distance of $\sim 3 \AA$ from the HR-TEM image correspond to the 002 plane of crystal orientation in XRD analysis. According to temperature dependent $\mathrm{PL}$ studies performed for $\mathrm{MAPbBr}_{3}$ bulk films, it is reported that at room temperature the $\mathrm{MAPbBr}_{3}$ bulk film is in tetragonal phase ${ }^{52}$, there is phase transition from tetragonal to cubic phase at $320 \mathrm{~K}$. Due to similarities in $\mathrm{MAPbBr}_{3}$ and $\mathrm{FAPbBr}_{3}$ structures, we propose that the $\mathrm{FAPbBr}_{3}$ to be in tetragonal phase at room temperature. However, the $\mathrm{XRD}$ measurements and indexing performed at room temperature indicate the $\mathrm{FAPbBr}_{3} \mathrm{NC}$ film to be in cubic phase as the XRD peaks are indexed to cubic phase (tetragonal indexing for XRD measurements resulted in lattice constants $\mathrm{a}$ and $\mathrm{b}$ to be very similar). From temperature dependent PL studies performed on $\mathrm{MAPbBr}_{3}$ and assuming the phase transitions for $\mathrm{MAPbBr}_{3}$ and $\mathrm{FAPbBr}_{3}$ to be similar, we believe that it is difficult to differentiate between these two phases. 



Figure 3. (a) The normalized PL emission spectra under a pump fluence of $1.2 \mu \mathrm{J} \mathrm{cm}^{-2}$ at temperatures ranging from 180-360 K. The PL spectra are normalized at their maximum intensity. (b) The change in FWHM is shown as a function of inverse temperature and in the inset the shift in the PL emission peak is shown as a function of temperature. (c) The integrated PL intensity as a function of inverse temperature and the Arrhenius fit (solid line) for temperature range 180-360 K (d) The TRPL intensity and the fit along with the average lifetime (inset) is shown for temperature range of $180-360 \mathrm{~K}$.

Temperature dependent exciton dynamics studies are performed to extract exciton binding energy and to understand PL dynamics as a function of temperature for $\mathrm{FAPbBr}_{3} \mathrm{NC}$ thin films. The exciton binding energy is directly related to the absorption and emission of an optoelectronic material.

Figure 3(a) shows normalized PL spectra for the temperature range from $180-360 \mathrm{~K}$ for $\mathrm{FAPbBr}_{3} \mathrm{NC}$ thin film under an excitation pump fluence of $1.2 \mu \mathrm{J} \mathrm{cm}^{-2}$. We restrict our temperature dependent studies to $180 \mathrm{~K}$ as the low temperature limit due to experimental constraints and we believe below $180 \mathrm{~K}$ the low temperature $\mathrm{PL}$ exciton dynamics to be similar to the $\mathrm{MAPbBr}_{3}$ thin films reported earlier ${ }^{52}$. The FWHM increases as the temperature increases as shown in Fig. 3(b) and this is attributed to increased phonon interactions and phonon scattering ${ }^{53,54}$. The thin film PL at $180 \mathrm{~K}$ is narrower with FWHM of $18.6 \mathrm{~nm}$, while at $360 \mathrm{~K}$ the FWHM of the PL is $46.9 \mathrm{~nm}$. There is red shift in PL peak position with lowering temperature consistent with $\mathrm{FAPbBr}_{3}$ bulk thin films and other lead halide perovskites ${ }^{49,53,54}$, the PL peak is at $\sim 530 \mathrm{~nm}$ at room temperature and the PL peak shifts to $\sim 545 \mathrm{~nm}$ at $180 \mathrm{~K}$ as shown in the inset of Fig. 3(b). This is attributed to lattice strain ${ }^{53}$, contraction of the lattice and reorientation of crystallites in the lattice ${ }^{54}$. The integrated PL emission intensity (area under the PL peak at different temperatures) versus the inverse of temperature for $\mathrm{FAPbBr}_{3} \mathrm{NCs}$ and the Arrhenius fits in the temperature range $180-360 \mathrm{~K}$ is shown in Fig. 3(c).

We also observe significant reduction in the absolute PL intensity with increase in temperature as shown in Figure $\mathrm{S} 1$ of supplementary info. The nonradioactive recombination is thermally activated in $\mathrm{FAPbBr}_{3}$ and is increasing with the temperature. We can extract exciton binding energy of these nanocrystals by fitting the curve with following equation ${ }^{55}$,

$$
I(t)=\frac{I_{0}}{1+A e^{-\frac{E_{b}}{k_{B} T}}}
$$

where $\mathrm{I}_{0}$ is the emission intensity extrapolated at $0 \mathrm{~K}, \mathrm{E}_{\mathrm{b}}$ is the exciton binding energy, and $\mathrm{k}_{\mathrm{B}}$ is the Boltzmann constant.

We find that the exciton binding energy of $\mathrm{FAPbBr}_{3} \mathrm{NCs}$ we synthesized is roughly $170 \mathrm{meV}$ (Fig. 3(c)) which is much higher than the reported exciton binding energies of the bulk thin films ${ }^{49}$. We have measured the time-resolved PL spectra for FAPbBr ${ }_{3} \mathrm{NCs}$ in thin film and in solution as shown in Fig. 3(d) for temperature range $180-360 \mathrm{~K}$. The PL decay transients can be fitted with the bi-exponential of the form ${ }^{23}$, 


\begin{tabular}{|l|c|c|c|c|c|}
\hline Temp(K) & $\mathbf{A}_{\mathbf{1}}$ (A1/A1+A2 in \%) & $\mathbf{T}_{\mathbf{1}}$ (ns) & $\mathbf{A}_{\mathbf{2}}$ (A2/A1+A2 in \%) & $\mathbf{T}_{\mathbf{2}}$ (ns) & $\begin{array}{c}\text { T-Average } \\
\text { (Error) }\end{array}$ \\
\hline Thin film \\
\hline $\mathbf{1 8 0}$ & $22463(47.5 \%)$ & 1.1 & $24774.9(52.5 \%)$ & 8.21 & $4.8( \pm 0.05)$ \\
\hline $\mathbf{2 1 0}$ & $11886(40.9 \%)$ & 1.2 & $17169.8(59.1 \%)$ & 11.4 & $7.2( \pm 0.07)$ \\
\hline $\mathbf{2 4 0}$ & $7852.6(38.5 \%)$ & 0.97 & $12512.9(61.5 \%)$ & 11.9 & $7.7( \pm 0.07)$ \\
\hline $\mathbf{2 7 0}$ & $4264.7(34.1 \%)$ & 1.0 & $8228.3(65.9 \%)$ & 14.4 & $9.8( \pm 0.07)$ \\
\hline $\mathbf{3 0 0}$ & $2128.2(32 \%)$ & 0.99 & $4521.6(68 \%)$ & 14.6 & $10.2( \pm 0.11)$ \\
\hline $\mathbf{3 3 0}$ & $1042.7(31.6 \%)$ & 0.90 & $2252(68.4 \%)$ & 13.4 & $9.4( \pm 0.114)$ \\
\hline $\mathbf{3 6 0}$ & $591.3(32.8 \%)$ & 0.90 & $1208.1(67.2 \%)$ & 15.6 & $10.7( \pm 0.16)$ \\
\hline Solution (Room Temperature) & $0.18(20 \%)$ & 5.45 & $0.70(80 \%)$ & 69.29 & 56 \\
\hline Solution & \multicolumn{5}{|l}{} \\
\hline
\end{tabular}

Table 1. Summarizes the $\mathrm{FAPbBr}_{3} \mathrm{NC}$ film and solution $\mathrm{PL}$ decay lifetimes and amplitude values as a function of temperature.

$$
A(t)=A_{1} \exp \left(\frac{-t}{\tau_{1}}\right)+A_{2} \exp \left(\frac{-t}{\tau_{2}}\right)
$$

For both thin film and solution, we observe bi-exponential decay resulting in two time constants one with the shorter lifetime, which we attribute to NCs whose recombination is strongly mediated by surface impurities and traps and the longer lifetime due to intrinsic band edge emission. The relative contribution of these two terms to the PL emission in solution shows that the fast component has only $20 \%$ contribution of the total PL emission and the faster lifetime component is $\sim 5 \mathrm{~ns}$. The rest $80 \%$ contribution of the PL emission is due to band edge emission and the corresponding slower component lifetime is $\sim 69 \mathrm{~ns}$. The TRPL for NCs in solution is shown in supplementary info Figure S3. For NC thin films, the faster component proportion roughly doubles in comparison to the solution sample from $20 \%$ to $32 \%$ at room temperature. The surface of the NCs is exposed in case of thin films and the surface trap density is much higher than in the case of the solution. The shorter lifetime $\tau_{1}$ is decreasing with increasing temperatures due to possible non-radiative recombination. The slower component proportion is reduced from $80 \%$ in solution to $\sim 70 \%$ in $\mathrm{NC}$ thin films at room temperature. The longer component $\tau_{2}$ increases with increasing temperature a similar to the trend that is observed in CdSe nano sheets, which is attributed to the giant oscillator strength (GOST) effect in low dimensions ${ }^{56}$. Although the average lifetime increases as a function of increasing temperature as shown in Table 1 and the inset of Fig. 3(d), the absolute PL signal intensity significantly decreases (shown in Figure S1 of supplementary info). This implies the PL quantum yield decreases as a function of increasing temperature, which is consistent with previous report in perovskite bulk films ${ }^{57}$. The average lifetime of $\mathrm{FAPbBr}_{3} \mathrm{NCs}$ film is much shorter in comparison to the solution sample, at room temperature NC thin film has average lifetime of $10 \mathrm{~ns}$ with PLQE roughly $25 \%$. The solution of $\mathrm{FAPbBr}_{3}$ in toluene on the other hand has much larger average lifetime of $55 \mathrm{~ns}$ and also much higher PLQE of 55-65\%.

We can estimate the exciton Bohr radius for these $\mathrm{FAPbBr}_{3} \mathrm{NC}$ system from the following equation,

$$
a_{b}=\frac{\hbar^{2} \varepsilon}{\mu e^{2}}
$$

where $\varepsilon$ is the static dielectric constant, $\hbar$ is the Planck's constant $1.05 * 10^{-34} \mathrm{Js}, \mu$ is the reduced exciton effective mass (the bulk value $0.13 \mathrm{~m}_{0}^{58}$ ) and e is unit charge of electron $1.6 * 10^{-19} \mathrm{Js}$ coulomb. The perovskite NC film is not dense in comparison to bulk perovskite film and hence the static dielectric constant value will be lower in comparison to bulk. The estimated Bohr radius is dependent on the value of the static dielectric constant. The exciton is also screened by phonons and random distribution of polar organic cation hence the value of $\varepsilon$ is not easy to estimate in these films. With $\varepsilon$ of $\sim 20$ (for $\mathrm{MAPbI}_{3}$ bulk films the static $\varepsilon$ is reported to be in the range of $35-70^{59}$ ), we estimate the Bohr exciton radius to be $8 \mathrm{~nm}$ much larger than the lattice constant. We do not observe the strong quantum confinement effect in these nanocrystals as a function of size due to their relatively larger size. This observation is supported by the PL emission measurements of supernatant (having nanocrystals of smaller size) and the precipitate (having nanocrystals of much larger size compared to supernatant), their emission maxima does not have strong shift as shown in supporting info Figure S2.

We fabricate the Pe-LED device with these $\mathrm{FAPbBr}_{3}$ NCs. The device structure and the flat band energy level diagram is shown in Fig. 4(a). The Pe-LED device with FAPbBr 3 perovskite NCs consists of $40 \mathrm{~nm}$ thick poly(ethylenedioxythiophene):polystyrene (PEDOT:PSS) as hole transport/injection layer, $\mathrm{FAPbBr}_{3} \mathrm{NC}$ layer and $\sim 50 \mathrm{~nm}$ of B3PYMPM or TPBi as an electron transport layer with $\mathrm{CsCO}_{3}(5 \mathrm{~nm}) / \mathrm{Al}(100 \mathrm{~nm})$ cathode layer. To observe any discontinuity in perovskite film and to see the thin film morphology, we perform the cross-sectional TEM imaging of the complete Pe-LED device. Figure S5 of Supplementary Information shows a cross-sectional TEM image of the Pe-LED device, with ITO and aluminum layers clearly identified. The HTL, perovskite EML and ETL in the device stack are not resolved as there is insufficient scattering contrast between them as there is dominant carbon content in all these films. The energy levels for the $\mathrm{FAPbBr}_{3} \mathrm{NC}$ layer are derived from UPS measurement shown in Figure S4 (Supplementary Information) and the energy level values for indium tin oxide (ITO) acting as semi-transparent electrode, PEDOT:PSS hole injection/transport layer (HIL/HTL), B3PYMPM and TPBi 

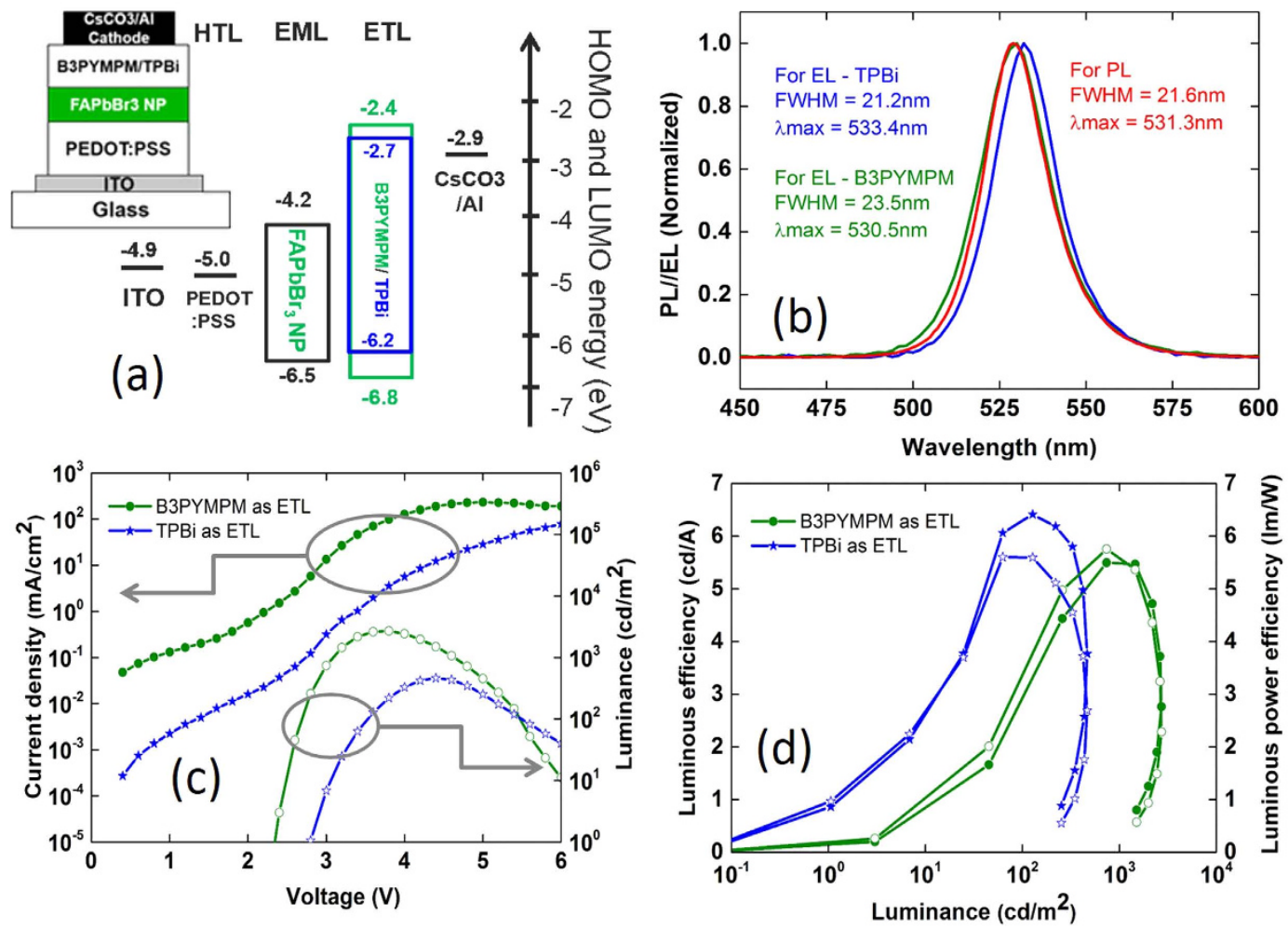

Figure 4. (a) The device structure for the Pe-LED device and the corresponding schematic energy level diagram for the materials. (b) EL spectra for Pe-LED device with B3PYMPM (green line) and TPBi (blue line) as ETL are shown in Fig. 4 recorded at $0.5 \mathrm{~mA} / \mathrm{cm}^{2}$. The PL spectrum of $\mathrm{FAPbBr}_{3}$ (red line is also shown for reference). (c) Current density/Luminance vs Voltage (J-V-L) characteristics for Pe-LED device with B3PYMPM (green line circles) and TPBi (blue line stars). (d) Luminous efficiency (cd/A) vs luminance $\left(\mathrm{cd} / \mathrm{m}^{2}\right)$ and Luminous power efficiency $(\mathrm{lm} / \mathrm{W})$ vs luminance $\left(\mathrm{cd} / \mathrm{m}^{2}\right)$ characteristics for Pe-LED device with B3PYMPM (green line circles) and TPBi (blue line stars). The filled symbols correspond to luminous efficacy $\mathrm{cd} / \mathrm{A}$ and open symbols correspond to $\mathrm{lm} / \mathrm{W}$.

electron transport layer (ETL) are taken from refs 20 and 60. The normalized EL spectra of the perovskite LED device with TPBi and B3PYMPM as ETL, the normalized PL spectrum of $\mathrm{FAPbBr}_{3} \mathrm{NC}$ film is shown in Fig. 4(b). The EL emission is due to radiative recombination of excitons in $\mathrm{FAPbBr}_{3} \mathrm{NCs}$ as the EL and PL spectra overlap and the small shift in the EL spectra for Pe-LED with TPBi layer is due to optical detuning of cavity from emission maxima. This is evident from the change in the EL spectra with the change in ETL.

The refractive index of B3PYMPM and TPBi are slightly different, hence for the device with the same thickness of ETL while keeping the other layers/thicknesses same results in an EL emission which is slightly red shifted. The FWHM of the EL spectrum is slightly broader in comparison to the PL spectra of neat FAPbBr $\mathrm{P}_{3}$ thin film. The $\mathrm{FAPbBr}_{3}$ PL emission has a FWHM of $22.5 \mathrm{~nm}$ with $\sim \lambda_{\max }$ of $530 \mathrm{~nm}$. The EL emission spectra of the Pe-LED device with TPBi as ETL has a FWHM of $21.2 \mathrm{~nm}$ with $\sim \lambda_{\max }$ of $533.4 \mathrm{~nm}$ while the Pe-LED device with B3PYMPM as ETL has a FWHM of $23.5 \mathrm{~nm}$ with $\sim \lambda_{\max }$ of $530.7 \mathrm{~nm}$. The current density-voltage-luminance curve for Pe-LED devices with TPBi and B3PYMPM as ETL layers is shown in Fig. 4(c). The turn-on voltages (corresponds to luminance of $1 \mathrm{~cd} \mathrm{~m}^{-2}$ ) are 2.8 and $2.3 \mathrm{~V}$ for Pe-LED devices with TPBi and B3PYMPM ETLs.

The luminance-voltage plot is similar for both ETLs. Initially, the luminance increases as a function of increasing voltage until $465 \mathrm{~cd} \mathrm{~m}^{-2}$ at $4.4 \mathrm{~V}$ (for TPBi ETL) and $2714 \mathrm{~cd} \mathrm{~m}^{-2}$ at $3.8 \mathrm{~V}$ (for B3PYMPM ETL) beyond the luminance maximum the increase in voltage results in decrease of luminance. The B3PYMPM ETL has superior electron mobility and better energetic alignment ${ }^{61}$, hence higher current densities in the Pe-LED device therefore resulting in higher luminance. The deeper HOMO of the B3PYMPM also helps in tighter exciton confinement, hence the device with B3PYMPM has a lower onset voltage. The Pe-LED device with TPBi as ETL has lower current density, lower luminance and superior efficiency values. The luminous and luminous power efficiency as a function of the luminance is shown in Fig. 4(d).

We achieve maximum luminous efficiency of $6.4 \mathrm{~cd} \mathrm{~A}^{-1}$ and a maximum luminous power efficiency of $5.6 \mathrm{lmW}^{-1}$ for the Pe-LED device with TPBi as ETL. For the Pe-LED device with B3PYMPM as ETL, we achieve a maximum current efficiency of $5.4 \mathrm{~cd} \mathrm{~A}^{-1}$ and a maximum luminous efficiency of $5.7 \mathrm{lmW} \mathrm{m}^{-1}$. There is a severe roll off in luminance and device efficiency beyond $3.8 \mathrm{~V}\left(98 \mathrm{~mA} / \mathrm{cm}^{2}\right)$ or the maximum luminance of $2714 \mathrm{~cd} / \mathrm{m}^{2}$ for the device with B3PYMPM as ETL and $4.8 \mathrm{~V}\left(16 \mathrm{~mA} / \mathrm{cm}^{2}\right)$ or the maximum luminance of $434 \mathrm{~cd} / \mathrm{m}^{2}$ for the device with TPBi as ETL. The Pe-LED device with both B3PYMPM and TPBi has a charge imbalance evident from the increase in efficiency until there is charge balance in the device. The sudden roll-off thereafter is severe 


\begin{tabular}{|l|c|c|c|c|}
\hline Voltage (V) & Current density $\left(\mathbf{m A} / \mathbf{c m}^{2}\right)$ & Luminance $\left(\mathbf{c d} / \mathbf{m}^{2}\right)$ & Cd/A & $\mathbf{l m} / \mathbf{W}$ \\
\hline QD LED device \\
\hline 6.6 & 0.54 & 92 & 17.1 & 8.1 \\
\hline 7.6 & 6.46 & 1357 & 21.0 & 8.1 \\
\hline 8.8 & 49.12 & 10550 & 21.4 & 7.6 \\
\hline \multicolumn{5}{|l|}{ Perovskite LED with FAPbBr ${ }_{3} \mathrm{NCs}$} \\
\hline 2.8 & 5.8 & 258 & 4.4 & 4.9 \\
\hline 3.8 & 98.3 & 2714 & 2.7 & 2.2 \\
\hline
\end{tabular}

Table 2. Summarizes the device performance values for QD LED device and the Pe-LED device.

and this roll off is similar in Pe-LED devices with different ETLs. The difference in the magnitude of the current density values (higher current density with B3PYMPM and lower current density with TPBi) does not have an influence on the quenching and efficiency roll off. The PLQE measured as a function of input laser power shown in Supplementary info Figure S7, for perovskite thin film has roughly same value. Implying the exciton quenching due to higher exciton density is less significant in Pe-LED devices. Hence, the charge injection seems to result in degradation of perovskite material ultimately causing the efficiency roll off. We compare typical current density, luminance and efficiency values for a quantum dot light emitting device (QD-LED) with similar device architecture except the difference in emission layer. The quantum dots have similar PLQE values ranging from 65-70\%. The device performance values for both QD-LED device and the Pe-LED device are tabulated in Table 2 at 100, 1000 and $10000 \mathrm{~cd} / \mathrm{m}^{2}$. The QD-LED device shows much higher luminance and efficiency values at lower current density in comparison to Pe-LED device. Hence, we believe better material engineering is essential to achieve higher efficiency in perovskite NC LED devices, our results clearly demonstrate that with the better perovskite material having lesser PL quenching at room temperature it should be possible to achieve much higher efficiency in Pe-LED devices.

\section{Conclusions}

In conclusion, we have demonstrated highly reproducible room temperature synthesis of device grade high quality formamidinium lead bromide $\left(\mathrm{FAPbBr}_{3}\right.$ ) colloidal nanocrystals (NC). The perovskite NC synthesized have high PLQE of 55-65\% and have very narrow FWHM of $\sim 20 \mathrm{~nm}$ suitable for display applications with high color purity. We also demonstrate high brightness perovskite light emitting device (Pe-LED) with these $\mathrm{FAPbBr}_{3}$ perovskite NC thin film using TPBi and B3PYMPM as electron transport layers (ETL). The Pe-LED device with TPBi as ETL has bright electroluminescence of up to $2714 \mathrm{~cd} / \mathrm{m}^{2}$, while the Pe-LED device with B3PYMPM as ETL as higher peak luminous efficiency of $6.4 \mathrm{~cd} / \mathrm{A}$ and peak luminous power efficiency of $5.7 \mathrm{~lm} / \mathrm{W}$. To our knowledge this is the first report on high brightness light emitting device based on $\mathrm{FAPbBr}_{3}$ nanocrystals in literature. Our studies indicate that the roll off in efficiency and the low performance in perovskite NC LED devices is attributed to the lattice changes due to charge injection and material degradation of the perovskite film. Hence, we believe better material engineering is essential to achieve higher efficiency in perovskite NC LED devices, our results clearly demonstrate that with the better perovskite material having lesser PL quenching at room temperature it should be possible to achieve much higher efficiency in Pe-LED devices.

\section{Methods}

Synthesis of $\left(\mathrm{CH}\left(\mathrm{NH}_{2}\right)_{2} \mathrm{PbBr}_{3}\right.$ or FAPbBr${ }_{3} \mathrm{NCs}$. $0.1 \mathrm{mmol}$ lead(II) bromide from Sigma Aldrich $(36.7 \mathrm{mg})$ and $0.1 \mathrm{mmol}$ formamidinium bromide from Dyesol $(25.6 \mathrm{mg})$ is dissolved in $0.5 \mathrm{~mL}$ of DMF which we name it as precursor solution. In a beaker $5 \mathrm{~mL}$ toluene, $10 \mu \mathrm{L}$ octylamine, $0.5 \mathrm{~mL}$ of oleic acid and $2 \mathrm{~mL}$ of butanol are added sequentially in that order and mixed on a magnetic stirrer at $800 \mathrm{rpm}$ at room temperature. To this solution $150 \mu \mathrm{L}$ of the precursor solution is added dropwise. The NCs are formed instantaneously, it is confirmed by color change - the transparent toluene solution turns bright yellowish green in color. Purification stage consists of 2 centrifuge steps. First centrifugation is performed at $7500 \mathrm{rpm}$ for 5 minutes, this will remove all the unreactive materials in the supernatant and the supernatant is discarded and the bottom precipitate is used for further purification. $2 \mathrm{~mL}$ toluene is added for another centrifugation at $7500 \mathrm{rpm}$ for $5 \mathrm{~min}$. This time the supernatant is transferred to vial for further use in device fabrication.

Perovskite film characterization. XRD data are collected on a Bruker Advance D8 X-ray diffractometer, using $\mathrm{Cu}-\mathrm{K} \alpha$ radiation, at a scanning rate of $0.01^{\circ}$ per step. X-ray photoelectron spectroscopy (XPS) and ultraviolet photoelectron spectroscopy (UPS) measurements are performed on the drop cast NC samples in UHV system. The XPS source is monochromatic $\mathrm{Al} \mathrm{K \alpha}$ with photon energy at $1486.7 \mathrm{eV}$. The UPS source is from a helium discharge lamp $(\mathrm{h} \nu=21.2 \mathrm{eV})$. The emitted photoelectrons are measured via an electron analyser (Omicron EA125). Small Angle X-ray Scattering (SAXS) experiments are performed using a SAXSess camera (Anton Paar, Graz, Austria) with a Cu anode X-ray source (PANalytical, PW3830) operating at $40 \mathrm{kV}$ and $50 \mathrm{~mA}$. Using a Goebel mirror and a collimation block the divergent polychromatic X-ray beam is collimated to a line-shaped beam of $\mathrm{Cu} \mathrm{K} \alpha$ radiation $(\mathrm{l}=0.154 \mathrm{~nm})$. The perovskite nanocrystals are dispersed in toluene and the solution is held in a quartz capillary holder. The experiment is performed at room temperature and the scattering pattern is collected on an imaging plate. Raw data processing (Integration and background subtraction) is performed using SAXSQuant software from Anton-Paar. The SAXS data is evaluated using an indirect Fourier transform (GIFT software from Anton-Paar) to obtain the pair distance distribution function (PDDF) $p(r)$, which can be used to 
determine the overall size and shape of the dispersed perovskite nanocrystals. TEM images are recorded on JEOL $2100 \mathrm{~F}$ advanced field emission microscope operating at $200 \mathrm{kV}$ accelerating voltage. The solution absorption is measured using Shimadzu spectrophotometer. The PL spectra are recorded with RF-5301PC fluorescence spectrometer (SHIMADZU). PLQE is measured using an integrated sphere and a fiber spectrometer (Ocean Optics USB 4000). The excitation source is $405 \mathrm{~nm}$ blue laser (Cobolt $\mathrm{MLD}^{\mathrm{TM}}$ ). Temperature dependent steady state photoluminescence (PL) and time-resolved PL (TRPL) are performed with a Coherent Libra ${ }^{\mathrm{TM}}$ femtosecond regenerative amplifier $(1 \mathrm{kHz}, \sim 50 \mathrm{fs})$ with frequency doubled $400 \mathrm{~nm}$ pulses at a pump fluence of $1.2 \mu \mathrm{J} \mathrm{cm} \mathrm{cm}^{-2}$ per pulse as the excitation source. The PL emission is collected in a conventional backscattering geometry and detected by a charge-coupled device array (Princeton Instruments, Pixis ${ }^{\mathrm{TM}}$ ) coupled to a monochromator (Acton, Spectra Pro $\left.{ }^{\mathrm{TM}}\right)$. The temporal evolution of the PL is resolved by an Optronis Optoscope ${ }^{\mathrm{TM}}$ streak camera system.

Device fabrication and characterization. ITO coated glass substrates with $10 \mathrm{ohm} / \mathrm{square}$ sheet resistance are purchased from Xiamen Weihua solar Co Ltd China. The substrates are sequentially washed with acetone, soap solution, ethanol and deionized water, followed by plasma treatment for $10 \mathrm{~min}$ at $100 \mathrm{~W}$ before use. The poly(3,4-ethylenedioxythiophene):poly(styrene-sulfonate) (PEDOT:PSS) Clevios Al 4043 Heraus $\mathrm{GmbH}$ solution is spin coated on the ITO film at $4000 \mathrm{rpm}$ for 60 seconds, then it is annealed at $150{ }^{\circ} \mathrm{C}$ for $15 \mathrm{~min}$. Then $\mathrm{FAPbBr}_{3}$ colloidal nanocrystals dispersed in toluene is spin-coated on the PEDOT:PSS film, followed by thermal annealing at $50^{\circ} \mathrm{C}$ for $1 \mathrm{~min}$. The substrates are then transferred to high vacuum thermal evaporator chamber, where 4,6-Bis(3,5-di(pyridin-3-yl)phenyl)-2-methylpyrimidine (B3PYMPM) or $2,2^{\prime}, 2^{\prime \prime}$-(1,3,5-Benzinetriyl)-tris(1-phenyl-1-H-benzimidazole) TPBi $(40 \mathrm{~nm})$, Caesium carbonate $\left(\mathrm{CsCO}_{3}\right)$ $(5 \mathrm{~nm})$ and Aluminium $(\mathrm{Al})(80 \mathrm{~nm})$ are sequentially thermally deposited. The active area of the devices fabricated in this work are $3 \mathrm{~mm}^{2}$. Devices are encapsulated before characterization.

The current-voltage-luminance characteristics is recorded via computer controlled source measure unit (Yokogawa GS610) and Konica Minolta LS-110 Luminance Meter. For measuring EQE of the LED device, Yokogawa GS610 source measure unit linked to a calibrated silicon photodiode is used to measure the currentvoltage-luminance characteristics and the electroluminescence spectra of the LED device are recorded via a spectrophotometer PR-655 (Photo Research, Inc.). The lifetime test is performed via photodiode placed on top of the LED device and the photodiode is controlled via a source measure unit in a constant current mode.

\section{References}

1. D’Innocenzo, V. et al. Excitons versus free charges in organo-lead tri-halide perovskites. Nat Commun 5, 3586, doi: 10.1038/ ncomms4586 (2014).

2. Zheng, F., Saldana-Greco, D., Liu, S. \& Rappe, A. M. Material Innovation in Advancing Organometal Halide Perovskite Functionality. J Phys Chem Lett 6, 4862-4872, doi: 10.1021/acs.jpclett.5b01830 (2015).

3. Nedelcu, G. et al. Fast Anion-Exchange in Highly Luminescent Nanocrystals of Cesium Lead Halide Perovskites $(\mathrm{CsPbX3}, \mathrm{X}=\mathrm{Cl}$, Br, I). Nano Lett 15, 5635-5640, doi: 10.1021/acs.nanolett.5b02404 (2015).

4. Saba, M., Quochi, F., Mura, A. \& Bongiovanni, G. Excited State Properties of Hybrid Perovskites. Acc Chem Res 49, 166-173, doi: 10.1021/acs.accounts.5b00445 (2016).

5. Even, J. et al. Solid-State Physics Perspective on Hybrid Perovskite Semiconductors. The Journal of Physical Chemistry C 119, 10161-10177, doi: 10.1021/acs.jpcc.5b00695 (2015).

6. Stranks, S. D. \& Snaith, H. J. Metal-halide perovskites for photovoltaic and light-emitting devices. Nat Nanotechnol 10, 391-402, doi: 10.1038/nnano.2015.90 (2015).

7. Berry, J. et al. Hybrid Organic-Inorganic Perovskites (HOIPs): Opportunities and Challenges. Adv Mater 27, 5102-5112, doi: 10.1002/adma.201502294 (2015).

8. Saparov, B. \& Mitzi, D. B. Organic-Inorganic Perovskites: Structural Versatility for Functional Materials Design. Chem Rev 116, 4558-4596, doi: 10.1021/acs.chemrev.5b00715 (2016).

9. Gratzel, M. The light and shade of perovskite solar cells. Nat Mater 13, 838-842, doi: 10.1038/nmat4065 (2014).

10. Liu, M., Johnston, M. B. \& Snaith, H. J. Efficient planar heterojunction perovskite solar cells by vapour deposition. Nature 501, 395-398, doi: 10.1038/nature12509 (2013)

11. Snaith, H. J. Perovskites: The Emergence of a New Era for Low-Cost, High-Efficiency Solar Cells. The Journal of Physical Chemistry Letters 4, 3623-3630, doi: 10.1021/jz4020162 (2013).

12. Green, M. A., Ho-Baillie, A. \& Snaith, H. J. The emergence of perovskite solar cells. Nature Photonics 8, 506-514, doi: 10.1038/ nphoton.2014.134 (2014).

13. Lee, M. M., Teuscher, J., Miyasaka, T., Murakami, T. N. \& Snaith, H. J. Efficient Hybrid Solar Cells Based on Meso-Superstructured Organometal Halide Perovskites. Science 338, 643-647 (2012).

14. Jeon, N. J. et al. Compositional engineering of perovskite materials for high-performance solar cells. Nature 517, 476-480, doi: $10.1038 /$ nature 14133 (2015).

15. Yang, W. S. et al. High-performance photovoltaic perovskite layers fabricated through intramolecular exchange. Science 348, 1234-1237, doi: 10.1126/science.aaa9272 (2015).

16. Han, Q. et al. Single Crystal Formamidinium Lead Iodide (FAPbI3): Insight into the Structural, Optical, and Electrical Properties. Adv Mater 28, 2253-2258, doi: 10.1002/adma.201505002 (2016).

17. Eperon, G. E. et al. Formamidinium lead trihalide: a broadly tunable perovskite for efficient planar heterojunction solar cells. Energy \& Environmental Science 7, 982, doi: 10.1039/c3ee43822h (2014).

18. D’Innocenzo, V., Srimath Kandada, A. R., De Bastiani, M., Gandini, M. \& Petrozza, A. Tuning the light emission properties by band gap engineering in hybrid lead halide perovskite. J Am Chem Soc 136, 17730-17733, doi: 10.1021/ja511198f (2014).

19. Huang, H. et al. Emulsion Synthesis of Size-Tunable $\mathrm{CH}_{3} \mathrm{NH}_{3} \mathrm{PbBr}_{3}$ Quantum Dots: An Alternative Route toward Efficient LightEmitting Diodes. ACS Appl Mater Interfaces 7, 28128-28133, doi: 10.1021/acsami.5b10373 (2015).

20. Zhang, X. et al. Enhancing the Brightness of Cesium Lead Halide Perovskite Nanocrystal Based Green Light-Emitting Devices through the Interface Engineering with Perfluorinated Ionomer. Nano Lett 16, 1415-1420, doi: 10.1021/acs.nanolett.5b04959 (2016).

21. Wong, A. B. et al. Growth and Anion Exchange Conversion of CH3NH3PbX3 Nanorod Arrays for Light-Emitting Diodes. Nano Lett 15, 5519-5524, doi: 10.1021/acs.nanolett.5b02082 (2015).

22. Zhang, F. et al. Brightly Luminescent and Color-Tunable Colloidal CH3NH3PbX3 (X = Br, I, Cl) Quantum Dots: Potential Alternatives for Display Technology. Acs Nano 9, 4533-4542 (2015). 
23. Li, X. et al. CsPbX3Quantum Dots for Lighting and Displays: Room-Temperature Synthesis, Photoluminescence Superiorities, Underlying Origins and White Light-Emitting Diodes. Advanced Functional Materials, n/a-n/a, doi: 10.1002/adfm.201600109 (2016).

24. Ling, Y. et al. Bright Light-Emitting Diodes Based on Organometal Halide Perovskite Nanoplatelets. Adv Mater 28, 305-311, doi: 10.1002/adma.201503954 (2016).

25. Tan, Z. K. et al. Bright light-emitting diodes based on organometal halide perovskite. Nat Nanotechnol 9, 687-692, doi: 10.1038/ nnano.2014.149 (2014).

26. Li, G. et al. Efficient light-emitting diodes based on nanocrystalline perovskite in a dielectric polymer matrix. Nano Lett 15, 2640-2644, doi: 10.1021/acs.nanolett.5b00235 (2015).

27. Sadhanala, A. et al. Electroluminescence from Organometallic Lead Halide Perovskite Conjugated Polymer Diodes. Advanced Electronic Materials 1 (2015).

28. Wang, J. et al. Interfacial Control Toward Efficient and Low Voltage Perovskite Light Emitting Diodes. Advanced Materials 27, 2311-2316 (2015).

29. Hoye, R. L. et al. Enhanced Performance in Fluorene Free Organometal Halide Perovskite Light Emitting Diodes using Tunable, Low Electron Affinity Oxide Electron Injectors. Advanced Materials 27, 1414-1419 (2015).

30. Shi, Z. et al. High-performance planar green light-emitting diodes based on PEDOT:PSS/CH $\mathrm{CH}_{3} \mathrm{NH}_{3} \mathrm{PbBr}_{3} / \mathrm{ZnO}$ sandwiched structure. Nanoscale, doi: 10.1039/c6nr00818f (2016).

31. Kim, Y. H. et al. Multicolored organic/inorganic hybrid perovskite light-emitting diodes. Adv Mater 27, 1248-1254, doi: 10.1002/ adma.201403751 (2015).

32. Brenner, T. M., Egger, D. A., Kronik, L., Hodes, G. \& Cahen, D. Hybrid organic-inorganic perovskites: low-cost semiconductors with intriguing charge-transport properties. Nature Reviews Materials 1, 15007, doi: 10.1038/natrevmats.2015.7 (2016).

33. Akkerman, Q. A. et al. Tuning the Optical Properties of Cesium Lead Halide Perovskite Nanocrystals by Anion Exchange Reactions. J Am Chem Soc 137, 10276-10281, doi: 10.1021/jacs.5b05602 (2015).

34. Era, M., Morimoto, S., Tsutsui, T. \& Saito, S. Organic-inorganic heterostructure electroluminescent device using a layered perovskite semiconductor $\left(\mathrm{C}_{6} \mathrm{H}_{5} \mathrm{C}_{2} \mathrm{H}_{4} \mathrm{NH}_{3}\right)_{2} \mathrm{PbI}_{4}$. Applied Physics Letters 65, 676, doi: 10.1063/1.112265 (1994).

35. Hattori, T., Taira, T., Era, M., Tsutsui, T. \& Saito, S. Highly efficient electroluminescence from a heterostructure device combined with emissive layered-perovskite and an electron-transporting organic compound. Chem Phys Lett 254, 103-108 (1996).

36. Chondroudis, K. \& Mitzi, D. B. Electroluminescence from an organic-inorganic perovskite incorporating a quaterthiophene dye within lead halide perovskite layers. Chemistry of Materials 11, 3028-3030, doi: DOI 10.1021/cm990561t (1999).

37. Cho, H. C. et al. Overcoming the electroluminescence efficiency limitations of perovskite light-emitting diodes. Science 350, $1222-1225$ (2015).

38. Xing, G. et al. Low-temperature solution-processed wavelength-tunable perovskites for lasing. Nat Mater 13, 476-480, doi: 10.1038/ nmat3911 (2014)

39. Zhu, H. et al. Lead halide perovskite nanowire lasers with low lasing thresholds and high quality factors. Nat Mater 14, 636-642, doi: $10.1038 /$ nmat4271 (2015)

40. Fu, Y. et al. Nanowire Lasers of Formamidinium Lead Halide Perovskites and Their Stabilized Alloys with Improved Stability. Nano Lett 16, 1000-1008, doi: 10.1021/acs.nanolett.5b04053 (2016).

41. Chin, X. Y., Cortecchia, D., Yin, J., Bruno, A. \& Soci, C. Lead iodide perovskite light-emitting field-effect transistor. Nat Commun 6, 7383, doi: 10.1038/ncomms8383 (2015)

42. Gonzalez-Carrero, S., Galian, R. E. \& Perez-Prieto, J. Organic-inorganic and all-inorganic lead halide nanoparticles [Invited]. Opt Express 24, A285-A301, doi: 10.1364/OE.24.00A285 (2016).

43. Protesescu, L. et al. Nanocrystals of Cesium Lead Halide Perovskites (CsPbX(3), X $=\mathrm{Cl}, \mathrm{Br}$, and I): Novel Optoelectronic Materials Showing Bright Emission with Wide Color Gamut. Nano Lett 15, 3692-3696, doi: 10.1021/nl5048779 (2015).

44. Schmidt, L. C. et al. Nontemplate synthesis of $\mathrm{CH}_{3} \mathrm{NH}_{3} \mathrm{PbBr}_{3}$ perovskite nanoparticles. J Am Chem Soc 136, 850-853, doi: 10.1021/ ja4109209 (2014)

45. Kim, J. Y., Voznyy, O., Zhitomirsky, D. \& Sargent, E. H. 25th anniversary article: Colloidal quantum dot materials and devices: a quarter-century of advances. Adv Mater 25, 4986-5010, doi: 10.1002/adma.201301947 (2013).

46. Shirasaki, Y., Supran, G. J., Bawendi, M. G. \& Bulović, V. Emergence of colloidal quantum-dot light-emitting technologies. Nature Photonics 7, 13-23, doi: 10.1038/nphoton.2012.328 (2012).

47. Song, J. et al. Quantum Dot Light-Emitting Diodes Based on Inorganic Perovskite Cesium Lead Halides (CsPbX3 ). Adv Mater 27, 7162-7167, doi: 10.1002/adma.201502567 (2015).

48. Aygüler, M. F. et al. Light-Emitting Electrochemical Cells Based on Hybrid Lead Halide Perovskite Nanoparticles. The Journal of Physical Chemistry C 119, 12047-12054, doi: 10.1021/acs.jpcc.5b02959 (2015).

49. Fang, H.-H. et al. Photoexcitation dynamics in solution-processed formamidinium lead iodide perovskite thin films for solar cell applications. Light: Science \& Applications 5, e16056, doi: 10.1038/lsa.2016.56 (2015).

50. Arora, N. et al. Photovoltaic and Amplified Spontaneous Emission Studies of High-Quality Formamidinium Lead Bromide Perovskite Films. Advanced Functional Materials, n/a-n/a, doi: 10.1002/adfm.201504977 (2016).

51. Hanusch, F. C. et al. Efficient Planar Heterojunction Perovskite Solar Cells Based on Formamidinium Lead Bromide. J Phys Chem Lett 5, 2791-2795, doi: 10.1021/jz501237m (2014).

52. Milot, R. L., Eperon, G. E., Snaith, H. J., Johnston, M. B. \& Herz, L. M. Temperature-Dependent Charge-Carrier Dynamics in CH3NH3PbI3Perovskite Thin Films. Advanced Functional Materials 25, 6218-6227, doi: 10.1002/adfm.201502340 (2015).

53. Huang, L.-y. \& Lambrecht, W. R. L. Electronic band structure, phonons, and exciton binding energies of halide perovskites $\mathrm{CsSnCl}_{3}$, $\mathrm{CsSnBr}_{3}$, and $\mathrm{CsSnI}_{3}$. Phys Rev B 88, doi: 10.1103/PhysRevB.88.165203 (2013).

54. Yu, C. L. et al. Temperature dependence of the band gap of perovskite semiconductor compound CsSnI ${ }_{3}$. Journal of Applied Physics 110 (2011).

55. Wu, K. et al. Temperature-dependent excitonic photoluminescence of hybrid organometal halide perovskite films. Phys Chem Chem Phys 16, 22476-22481, doi: 10.1039/c4cp03573a (2014).

56. Achtstein, A. W. et al. Electronic structure and exciton-phonon interaction in two-dimensional colloidal CdSe nanosheets. Nano Lett 12, 3151-3157, doi: 10.1021/nl301071n (2012).

57. Stranks, S. D. et al. Recombination Kinetics in Organic-Inorganic Perovskites: Excitons, Free Charge, and Subgap States. Physical Review Applied 2, doi: 10.1103/PhysRevApplied.2.034007 (2014).

58. Tanaka, K. et al. Comparative study on the excitons in lead-halide-based perovskite-type crystals $\mathrm{CH}_{3} \mathrm{NH}_{3} \mathrm{PbBr}_{3} \mathrm{CH}_{3} \mathrm{NH}_{3} \mathrm{PbI}_{3}$. Solid State Commun 127, 619-623, doi: 10.1016/s0038-1098(03)00566-0 (2003).

59. Lin, Q., Armin, A., Nagiri, R. C. R., Burn, P. L. \& Meredith, P. Electro-optics of perovskite solar cells. Nature Photonics 9, 106-112, doi: 10.1038/nphoton.2014.284 (2014).

60. Perumal, A. et al. High Efficiency, Solution Processed, Multilayer Phosphorescent Organic Light Emitting Diodes with a Copper Thiocyanate Hole Injection/Hole Transport Layer. Advanced Materials 27, 93-100 (2015).

61. Sasabe, H. et al. 2-Phenylpyrimidine skeleton-based electron-transport materials for extremely efficient green organic light-emitting devices. Chem Commun (Camb), 5821-5823, doi: 10.1039/b812270a (2008) 


\section{Acknowledgements}

This research is supported by the National Research Foundation, Prime Minister's Office, Singapore under its competitive Research Programme (CRP Award No. NRF-CRP14-2014-03). H.V.D acknowledges the financial support from NRF Investigatorship grant NRF-NRFI2016-08. T.C.S. acknowledges the financial support from NTU start-up grant M4080514; the Ministry of Education AcRF Tier 1 grant RG101/15, and Tier 2 grants MOE2013-T2-1-081 and MOE2014-T2-1-044.

\section{Author Contributions}

A.P. and H.V.D. conceived the idea and designed the experiment. A.P. synthesized and prepared the samples for all characterizations, also A.P. fabricated and tested the LED devices which was supervised by H.V.D. S.S. assisted in LED device fabrication. M.L.J. and Y.K.T.E. carried out temperature dependent time resolved measurements and was supervised by T.C.S. Q.L. performed TEM cross-section and FIB measurements, was supervised by C.L.G. S.C. measured U.P.S. and X.P.S. scans. A.P. and H.V.D. together with all authors carried out data analysis. The manuscript is prepared through contributions of all authors. All authors have given approval to the final version of the manuscript.

\section{Additional Information}

Supplementary information accompanies this paper at http://www.nature.com/srep

Competing financial interests: The authors declare no competing financial interests.

How to cite this article: Perumal, A. et al. High brightness formamidinium lead bromide perovskite nanocrystal light emitting devices. Sci. Rep. 6, 36733; doi: 10.1038/srep36733 (2016).

Publisher's note: Springer Nature remains neutral with regard to jurisdictional claims in published maps and institutional affiliations.

(c) (i) This work is licensed under a Creative Commons Attribution 4.0 International License. The images or other third party material in this article are included in the article's Creative Commons license, unless indicated otherwise in the credit line; if the material is not included under the Creative Commons license, users will need to obtain permission from the license holder to reproduce the material. To view a copy of this license, visit http://creativecommons.org/licenses/by/4.0/

(c) The Author(s) 2016 